\title{
Identidades. Revista de Ciencias Sociales y Humanidades, año 5, núm. 9, 2016. San Salvador: Dirección General de Investigaciones en Cultura y Arte, Secretaría de Cultura de la Presidencia.
}

Miguel Lisbona-Guillén

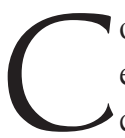

omo bien indica Ramón Rivas en el prólogo de este número de la revista, desde hace más de quince años la antropología se ha convertido en una disciplina con presencia visible en El Salvador. Y también que se ha institucionalizado a través de su enseñanza en la Universidad Nacional (2005) y en la Universidad Tecnológica de El Salvador (2000), ambas en la capital del país, San Salvador.

Para que lo anterior ocurriera fue fundamental la creación, en 1995, del Instituto de Estudios Históricos, Antropológicos y Arqueológicos de la Universidad de El Salvador. El impulso que tal dependencia otorgó a la investigación, con proyectos tales como los denominados "Memoria histórica del Movimiento Campesino de Chalatenango", "El empoderamiento de mujeres por participación: un estudio de campo de mujeres salvadoreñas en movimientos sociales" (Lara, 2011: 75), y otros preocupados por la emigración, abrieron el camino para que la antropología se considerara una disciplina de interés en instituciones tanto públicas, como privadas.

El Dr. Ramón Rivas, como el Dr. Carlos Lara, coordinador del último número de la revista, hacen hincapié, y con rotundidad, en la necesidad que esta disciplina social tiene, o debe tener, de comprometerse ineludiblemente con la realidad de su país. Las dificultades del mundo global, no cabe duda, también se hacen visibles en países centroamericanos como El Salvador, con una larga historia de desigualdades sociales, dominación política e injusticia generalizada. Este hecho ubica la antropología como una herramienta que transciende su condición de creadora de conocimientos y se ubica como un instrumento para comprender la realidad social, con el objetivo de transformar a esta última en beneficio de los ciudadanos, tanto de los que viven en su suelo como de los miles de salvadoreños, y de otros centroamericanos, que han emigrado allende sus fronteras territoriales.

De esta manera, es fácil comprender cómo los perfiles de las licenciaturas de Antropología Sociocultural en la Universidad de El Salvador y de Antropología en la Universidad Tecnológica de El Salvador, muestran en sus indicaciones que los conocimientos que adquieran los estudiantes los harán "capaces de proporcionar soluciones concretas a los problemas más agudos del país y del área centroamericana". Si lo anterior se menciona en la universidad pública, en la privada, que recientemente ha reestructurado su plan de estudios, se señala el deseo de mejorar "la calidad académica para responder a los desafíos y compromisos con la sociedad" y a "las necesidades de formación que demanda el país", todo ello con la

Miguel Lisbona Guillén, Universidad Nacional Autónoma de México, México. Correo electrónico: mlisbonag@hotmail.com.

Recibida: 23 de noviembre de 2017 
finalidad de "contribuir al desarrollo y a la solución de la problemática nacional". ${ }^{2}$ Es decir, en ambos casos se enfatiza, de manera notable e insistente, la necesidad que la disciplina antropológica tiene para apoyar el desarrollo del país y la construcción de alternativas reales de crecimiento, sin olvidar los principios éticos que acompañan la deontología antropológica, algo claramente especificado en la Universidad Tecnológica:

El Salvador es nuevo en instituciones de educación superior que formen profesionales en Antropología, pese a que el proceso de desarrollo integral del país lo demanda. Esta demanda se hace más urgente en esta época de principios de siglo, en que el país está atravesando una de las etapas más dinámicas de su historia, ante el reordenamiento mundial, cuyos cambios políticos, sociales, económicos y culturales requieren con urgencia la labor profesional de los antropólogos, especialmente para impulsar el desarrollo de la investigación sobre la cultura de las generaciones vivientes: sus valores, sus costumbres, sus modos de vivir y, en definitiva, su identidad cultural. Todo esto es necesario para poder integrarse como país y entre países, en el contexto de la globalización mundial. ${ }^{3}$

Y si ello es notorio en las dos instituciones que instruyen a profesionistas en El Salvador, no lo es menos la idea de promover la formación de investigadores que tengan como referente el carácter científico de la antropología, incluso con el objetivo loable, aunque nada fácil, de "impulsar la creación de una tradición de investigación antropológica en el país". ${ }^{4}$

Para quien no conozca las instituciones académicas donde se imparte esta ciencia social en el país centroamericano, dichos deseos o propósitos pueden parecer excesivos y sumamente ambiciosos; sin embargo, debería saber que, a pesar de las complejas condiciones económicas y de las siempre crecientes carencias, existe un compromiso diáfano de los docentes, y también de los alumnos, en la construcción de la disciplina y en el crecimiento en la cantidad y calidad de las investigaciones, entusiasmo que se quisiera a veces observar en otras instituciones públicas y privadas de muchos países donde la antropología se imparte o, incluso, tiene largo aliento histórico. Referencias para conocer la trayectoria de la enseñanza, o de la propia antropología en Centroamérica, son consultables en trabajos preocupados por la conformación de una tradición propia o, al menos, surgida de investigaciones que involucren a académicos locales: María Eugenia Bozzoli y Margarita Bolaños (2011), Margarita Bolaños y María Eugenia Bozzoli (2015) y Gabriel Ascencio (ed.) (2010).

Cabe mencionar que Ramón Rivas, quien presenta el número de la revista, es una figura indispensable para entender la tarea antropológica y docente desde la Universidad Tecnológica de El Salvador, aunque haya ocupado otros puestos académicos o como funcionario público en su país. Además de haberse formado como antropólogo en la universidad holandesa de Nijmegen, su trabajo de investigación trasciende a su lugar de origen; sobre todo destacan sus obras efectuadas sobre Honduras (1993) y El Salvador (2000) y, últimamente, la que realizó sobre la historia de la antropología en El Salvador (Rivas, 2012). Este texto inicia con los cronistas y viajeros, y abarca hasta las pesquisas más recientes que tienen como objeto de estudio el país centroamericano, pasando por aportaciones como las de literatos locales. Su interés en la investigación y la docencia le ha conducido, incluso, a afirmar la relevancia que tendría la enseñanza de la antropología en la educación básica y el bachillerato con el fin de que: "los estudiantes entiendan que lo que somos y hacemos en nuestra sociedad es producto de esa cultura en que vivimos [...]. La cultura se aprende, la cultura se trasforma y si queremos podemos hacer un país con una cultura para la sana convivencia".

Como ya mencioné, Carlos Lara es quien coordina este número, y lo abre repasando los procesos que experimenta o que debe caminar la antropología en su país, todo ello bajo el deseo de que "la investigación de campo" proporcione "los fundamentos teóricos para definir el tipo (o los tipos) de desarrollo que se debe generar en El Salvador". Cabe señalar que dicho académico tiene una larga trayectoria en la 
investigación (Lara, 1994, 2003 y 2006), y es pieza clave en la docencia desde la universidad pública. Junto a las investigaciones de campo, también ha reflexionado y discutido la historia de la antropología en su país, algo que desde la construcción institucional ya ofrecieron, a finales del siglo pasado, Ana Lillian Ramírez y América Rodríguez (1993).

En su artículo de revisión sobre el pasado más reciente de la investigación antropológica en El Salvador, Carlos Lara (2011) repasa las corrientes teóricas, así como los autores, que se han convertido en antecedentes de la disciplina en el país centroamericano. De ahí que tras mencionar las clásicas narraciones de los cronistas coloniales se dirija al periodo en el que se construye la propia antropología en todo el orbe. A finales del siglo XIX, y principios del XX, la embrionaria investigación tiene su origen en la presencia de académicos procedentes del norte de Europa dedicados a efectuar pesquisas en el occidente de El Salvador. Aunque su repercusión en el país fue muy tardía, o de poca trascendencia para conformar un referente científico local, fueron el sueco Carl Hartman y los alemanes Robert Lehman y Leonhard Schultz quienes se acercaron a las poblaciones indígenas con las lógicas limitaciones científicas y prejuicios del momento histórico. Este hecho lo resalta el propio Lara como un adeudo de tales investigaciones al no tener influencia para desarrollar una tradición propia (2011: 70).

Posteriores trabajos como los del estadounidense Richard N. Adams, en la mitad del siglo XX, que tuvieron como objeto de estudio todo el territorio centroamericano, tampoco impactaron en lo local, más bien el investigador salvadoreño considera que sólo se debe hablar del surgimiento de la ciencia antropológica en el país hasta mediados del siglo XX, cuando dos incipientes influencias se aprecian en suelo salvadoreño, la primera surgida de la escuela marxista, muy en boga en las décadas de los sesenta y setenta del siglo pasado, mientras que la otra se encuentra en el folklorismo (Lara, 2011: 71-72). Esta última propuesta fue tal vez tardía en El Salvador, desde mi perspectiva, pero coincidente con los apoyos o deseos surgidos desde los gobiernos autoritarios que dominaron el país.
En la primera influencia fueron imprescindibles los trabajos de Alejandro D. Marroquín $(1964,1974)$, convertidos en puntas de lanza de la antropología local. Él, como otros de sus sucesores, tuvo en México una referencia para su formación, además de ser un partícipe en las instituciones indigenistas del momento en suelo mexicano. Con respecto a El Salvador, publicó dos obras con la colaboración de alumnos de las cátedras de Sociología en municipios con presencia indígena (Lara, 2011: 71). Por su parte, los trabajos folkloristas de los años setenta del siglo pasado tomaron como referente las propuestas del guatemalteco Celso Lara, de amplia influencia en su país y en otros de la región, para asentar los valores nacionales y de la identidad en pasados remotos y a través de la conservación de prácticas y valores culturales considerados tradicionales (Lara, 2011: 72-73). Esta exploración efectuada por Carlos Lara Martínez le sirve para indicar que es en los inicios del siglo XXI cuando la arriba mencionada institucionalización de la docencia determinó, con certeza, la consolidación de "una antropología sociocultural salvadoreña", la cual ya se encuentra en disposición de poder evaluar las aportaciones que hicieron académicos extranjeros, además de efectuar investigaciones originales desde el propio país (Lara, 2011: 70). Estas indagaciones, eso sí, las divide en dos tendencias, una más descriptiva y otra interpretativa, esta última pensada como forma de analizar los procesos socioculturales de la sociedad salvadoreña actual (Lara, 2011: 75).

Tal preámbulo permite entender que el texto que ofrece Carlos Lara Martínez en el número de la revista tiene en la mira el presente y, sobre todo, el futuro de la disciplina en El Salvador. Ello le inclina a dividir su trabajo en cuatro partes que denomina "Integración de la teoría con la investigación de campo", "Perspectiva holística o totalizadora", "Aplicación del método etnográfico como método principal de la investigación” e "Integración de lo local con lo nacional y lo global".

El título de estos apartados habla por sí mismo de su intención, pero quiero destacar, por encima de todo, la idea que sitúa la investigación en El Salvador más allá de los localismos que, en ocasiones, tanto daño 
han hecho a nuestra disciplina. La visión del país en el mundo global, con las constantes transformaciones que ha sufrido en las últimas décadas, se convierte en algo ineludible. ¿Cómo no tomar en cuenta el flujo de los capitales económicos y las crisis que éstos pueden producir, los modelos de desarrollo, la emigración o la integración regional, a la hora de insertar cualquier pesquisa social en países como los centroamericanos? Éstos son los motivos por los que el artículo se convierte, junto al que revisó el pasado de la investigación antropológica en El Salvador, en un referente para alumnos e investigadores empeñados en la conformación de una tradición etnográfica propia fundamentada en la metodología clásica, pero incorporando las temáticas emergentes que atraviesan la realidad regional.

El artículo de Luis Rodríguez Castillo, investigador mexicano cada vez más vinculado con El Salvador, es el siguiente en el número. Después de la reflexión sobre la pesquisa académica, como experiencia personal a través de sus pasos como estudiante y académico, Rodríguez lanza propuestas sobre cómo ampliar los horizontes de la investigación sobre el poder, y sus formas y manifestaciones en el mundo actual. Estos replanteamientos epistemológicos y teóricos incluyen metodologías variadas para intentar dilucidar las complejidades de la realidad presente.

Se trata de reflexiones de largo aliento en la historia de la antropología puesto que unen la propia experiencia individual y de formación, con las situaciones observadas en el trabajo de campo para repensar el papel de la disciplina que, por su singular metodología, tiene en la convivencia con otros seres humanos el trasfondo de las interpretaciones y, por qué no decirlo, de los anhelos de cambio en situaciones que nos ponen en dilemas éticos o cuestionan posicionamientos políticos.

A estos textos le siguen los de noveles antropólogos, pero muy comprometidos, como afirmé arriba, con la investigación en su país. Ese grupo de artículos lo abre el de Adriana Alas con el título de "La dinámica de los grupos domésticos en una repoblación al oriente de Chalatenango, municipio Las Vueltas, Cantón La Ceiba (2011-2013)". Aunque replantear el orden expositivo y sus prioridades ayudaría a los lectores, el trabajo sugiere una propuesta de interés al engarzar aspectos teóricos con otros etnográficos e históricos. En tal sentido, profundizar en concepciones como la de "grupo doméstico" y su relación con lo que denomina "dinámica tradicional" es pertinente para que de la etnografía surja la necesaria interpretación. Es decir, resulta siempre complejo remitir situaciones a tiempos muy remotos si no se cuenta con la información pertinente, algo en lo que la antropología funcionalista cayó en demasiadas ocasiones.

"Transformación sociocultural en los Quebrachos, Jocoiatique, una visión desde la posguerra" es el trabajo presentado por Clara Guardado Torrez. En su texto ofrece una propuesta de interés por observar cómo se organizó una localidad tras el conflicto armado vivido en El Salvador durante el siglo pasado que provocó un sinnúmero de víctimas y desplazados. Sin embargo, desea abarcar muchos temas y aspectos de la realidad pasada y presente de la mencionada localidad, y ello puede ocasionar que disperse el valor real del trabajo. Lo anterior seguramente es debido a que el origen del trabajo se encuentra en otro más extenso en forma de tesis de licenciatura, y la división de esta última es posible que cause la mencionada dispersión.

Estanislao Enrique López escribe "Semana Santa en Sonsonate. El carácter sacrificial de un ritual comunitario". El artículo responde a uno de los temas clásicos de la antropología como es la descripción de un ritual religioso. En este sentido, el autor resalta las fases del ritual siguiendo las clásicas definiciones de Arnold Van Gennep y su relectura por parte de Victor Turner. Aquí radica uno de los aspectos nodales de su análisis que, como trabajo incipiente, debe incorporar textos bibliográficos para establecer comparaciones $\mathrm{u}$ otras visiones de manifestaciones religiosas con el mismo origen, como es la Semana Santa. De la misma forma, la introducción de elementos interpretativos, como la condición sacrificial de los sujetos de estudio participantes en el ritual, requeriría una mayor discusión.

El texto ganaría en precisión si omitiera ciertas afirmaciones que pueden ser dudosas o discutibles, me refiero a aquellas que hablan de la Semana Santa 
como un rito de sacrificio por excelencia o como un reflejo de "los valores de la cultura de los pueblos de Mesoamérica: sacrificio, muerte, dolor y sufrimiento". El contenido propio del cristianismo no difiere de lo expresado por el autor; además, los ritos de la Semana Santa tienen expresiones en diversos lugares del mundo que responderían a lo que en el texto se llama valores de Mesoamérica.

"La violencia pandilleril: más allá de la muerte" es el título otorgado por Juan José Martínez a un trabajo sumamente original por la temática expuesta. El contenido también ofrece innumerables aspectos de interés para conocer la organización, funcionamiento y expresión de las maras salvadoreñas. Igualmente, muestra osadía teórica para efectuar propuestas que hagan comprensible la violencia de individuos en el seno de tales organizaciones. Sin embargo, la concreción que debe tener un artículo hace que en algunos momentos se echen de menos discusiones antropológicas que tratan el tema de la violencia física y simbólica, lo que le permitiría ampliar su horizonte de análisis y ubicaría su trabajo de campo en discusiones de más largo aliento académico.

Este número de la revista acaba con la sección "Avances de investigación", donde un trabajo arqueológico sobre el primer poblamiento del país, con gran cantidad de información de campo, abre posibilidades para la continuidad de investigaciones del pasado en todo el Istmo centroamericano. Asimismo, existe otra sección denominada "Fuentes" y una última consistente en "Reseñas".

Para finalizar deseo destacar dos hechos que la aparición de este número de la revista me provoca como lector y, también, como interesado en la realidad centroamericana. En primer lugar, hay que saludar y aplaudir el esfuerzo que instituciones y personas concretas efectúan para que este proyecto editorial tenga continuidad. Es conocido que los gobiernos, y las instituciones como sus representantes visibles, no siempre consideran la labor en ciencias sociales prioritaria para el crecimiento de sus países, visto sólo desde el prisma económico. De ahí que el mantenimiento y la periodicidad de la revista signifiquen sobreponerse a adversidades ajenas, en muchas ocasiones, al compromiso de los académicos e investigadores de El Salvador.

Considerar los estudios antropológicos como una responsabilidad asumida como deber hacia su país y la región centroamericana es una cuestión no siempre comprendida por los políticos, pero que se hace presente en los documentos oficiales de las licenciaturas en Antropología de El Salvador cuando afirman que la disciplina facilita:

el entendimiento de grupos diversos al igual que la educación del pueblo, es una base incuestionable para potenciar el ejercicio de la democracia en cualquier país; por tanto, la labor de los antropólogos trasciende las tareas de tipo cultural y de conservación de bienes de patrimonio cultural, para contribuir al proceso de democratización e integración regional de los países. ${ }^{6}$

En segundo lugar, es loable la tarea llevada a cabo desde las aulas salvadoreñas para comprometer a sus alumnos en la realización de etnografía. Si algo singulariza a la disciplina antropológica es el trabajo de campo como su metodología primordial, y es ahí donde este número de la revista destaca puesto que ofrece partes de trabajos de titulación de noveles antropólogos locales, profesionistas que hacen de la experiencia de campo el sustento fundamental para la construcción de sus exposiciones y del contenido de sus análisis. Por tal motivo, la experiencia del número 9 de la revista Identidades se convierte en ejemplo nítido de publicación donde pueden foguearse los nuevos antropólogos, así como un espacio de divulgación de temas de interés para los países centroamericanos.

\section{Notas}

${ }^{1}$ Ver: https://bit.ly/2FWvG7v (consultado el 14 de marzo de 2017).

${ }^{2}$ Ver: https://bit.ly/1NgVW9q (consultado el 14 de marzo de 2017). 
${ }^{3}$ Ver: https://bit.ly/1NgVW9q (consultado el 14 de marzo de 2017).

${ }^{4}$ Ver: https://bit.ly/1NgVW9q (consultado el 14 de marzo de 2017).

5 Ver: https://bit.ly/2DLEWFx (consultado el 14 de marzo de 2017).

${ }^{6}$ Ver: https://bit.ly/1NgVW9q (consultado el 15 de marzo de 2017).

\section{Referencias}

Ascencio Franco, Gabriel (ed.) (2010). La antropología en Centroamérica. Reflexiones y perspectivas. México: Red Centroamericana de Antropología, Universidad de Ciencias y Artes de Chiapas, Universidad Nacional Autónoma de México, Universidad Intercultural de Chiapas.

Bolaños Arquín, Margarita y María Eugenia Bozzoli (2015). Introducción al estudio del desarrollo de la antropología centroamericana, 1880-2013. San José de Costa Rica: Universidad de Costa Rica.

Bozzoli, María Eugenia y Margarita Bolaños Arquín (2011). "La enseñanza de la antropología en Centroamérica: ¿desde lo propio o lo ajeno?" En Alteridades, 21(41), enero-junio: 107-110.

Lara Martínez, Carlos B. (1994). Salvadoreños en Calgary: el proceso de configuración de un nuevo grupo étnico. San Salvador: Dirección General del Patrimonio Cultural.
Lara Martínez, Carlos B. (2003). Joya de Cerén. La dinámica sociocultural de una comunidad semicampesina de El Salvador. San Salvador: CONCULTURA.

Lara Martínez, Carlos B. (2006). La población indígena de Santo Domingo de Guzmán. Cambio y continuidad sociocultural. San Salvador: CONCULTURA.

Lara Martínez, Carlos B. (2011). "El desarrollo de la antropología sociocultural en El Salvador". En Alteridades, 21(41): 69-98.

Marroquín, Alejandro Dagoberto (1964). San Pedro Nonualco. Investigación sociológica. San Salvador: Editorial Universitaria.

Marroquín, Alejandro Dagoberto (1974). Panchimalco. Investigación sociológica. San Salvador: Editorial Universitaria.

Ramírez,AnaLilliany América Rodríguez(1993). “Algunas reflexiones sobre el desarrollo de la antropología en El Salvador". En Cuadernos de Antropología, 9: 37-47. San José: Universidad de Costa Rica.

Rivas, Ramón D. (1993). Pueblos indígenas y garífuna de Honduras (una caracterización). Tegucigalpa: Editorial Guaymuras.

Rivas, Ramón D. (2000). Ilobasco: una aproximación histórica y antropológica. San Salvador: Universidad Tecnológica de El Salvador.

Rivas, Ramón D. (2012). AntropologíaenElSalvador: recorrido histórico y descriptivo. San Salvador: Universidad Tecnológica de El Salvador. 